\title{
Mendelian randomization analysis of vitamin $D$ in the secondary prevention of hypertensive-diabetic subjects: role of facilitating blood pressure control
}

\author{
Yap-Hang Chan ${ }^{1}$ (D, C. Mary Schooling², Jie V. Zhao², Shiu-Lun Au Yeung², Jo Jo Hai ${ }^{1,3}$, G. Neil Thomas', \\ Kar-Keung Cheng ${ }^{4}$, Chao-Qiang Jiang ${ }^{5}$, Yuen-Kwun Wong ${ }^{1}$, Ka-Wing Au ${ }^{1}$, Clara S. Tang ${ }^{6}$, Chloe Y. Y. Cheung ${ }^{7}$, \\ Aimin $\mathrm{Xu}^{7}$, Pak-Chung Sham ${ }^{6}$, Tai-Hing Lam ${ }^{2^{*}+}$, Karen Siu-Ling Lam ${ }^{7^{*+}}$ and Hung-Fat Tse ${ }^{1,3,8,9^{*+}}$
}

\begin{abstract}
Background: Vitamin D (Vit-D) promotes vascular repair and its deficiency is closely linked to the development of type 2 diabetes mellitus (T2DM) and hypertension. Whether genetially predicted vitamin D status (serological 25hydroxyvitamin $\mathrm{D}[25(\mathrm{OH}) \mathrm{D}]$ ) confers secondary protection against cardiovascular diseases (CVD) among high-risk hypertensive-diabetic subjects was unknown.

Methods: This is a prospective, individual-data, two-sample Mendelian randomization study. We interrogated 12 prior GWAS-detected SNPs of comprehensive Vit-D mechanistic pathways using high-throughput exome chip analyses in a derivation subcohort $(n=1460)$ and constructed a genetic risk score (GRS) (rs2060793, rs4588, rs7041; F-statistic $=32, P<$ 0.001) for causal inference of comprehensive CVD hard clinical endpoints in an independent sample of hypertensive subjects ( $n=3746$ ) with prevailing co-morbid T2DM $(79 \%)$ and serological $25(\mathrm{OH}) \mathrm{D}$ deficiency $[<20 \mathrm{ng} / \mathrm{mL}] 45 \%$.

Results: After $55.6 \pm 28.9$ months, 561 (15\%) combined CVD events including myocardial infarction, unstable angina, ischemic stroke, congestive heart failure, peripheral vascular disease, and cardiovascular death had occurred. KaplanMeier analysis showed that genetically predicted reduced vitamin $\mathrm{D}$ status was associated with reduced event-free survival from combined CVD events (log-rank $=13.5, P=0.001$ ). Multivariate-adjusted per-allele increase in GRS predicted reduced combined CVD events ( $H R=0.90$ [0.84 to 0.96], $P=0.002$ ). Mendelian randomization indicates that increased Vit-D exposure, leveraged through each $1 \mathrm{ng} / \mathrm{mL}$ genetically instrumented rise of serum Vit-D, protects against combined CVD events (Wald's estimate: $\mathrm{OR}=0.86$ [95\% Cl 0.75 to 0.95$]$ ), and myocardial infarction ( $\mathrm{OR}=0.76$ [ $95 \% \mathrm{Cl} 0.60$ to 0.90$]$ ]). Furthermore, genetically predicted increase in Vit-D status ameliorates risk of deviation from achieving guideline-directed hypertension control (JNC-8: systolic target $<150 \mathrm{mmHg})(\mathrm{OR}=0.89$ [95\%Cl 0.80 to 0.96$])$.
\end{abstract}

\footnotetext{
*Correspondence: hrmrlth@hku.hk; ksllam@hku.hk; hftse@hkucc.hku.hk

${ }^{\dagger}$ TH Lam, KSL Lam, and HF Tse contributed equally to the supervision of this work and are co-corresponding authors.

${ }^{2}$ School of Public Health, The University of Hong Kong, Hong Kong, SAR,

China

${ }^{7}$ Division of Endocrinology, Queen Mary Hospital, The University of Hong

Kong, Hong Kong, China

'Division of Cardiology, Queen Mary Hospital, The University of Hong Kong,

Hong Kong, China

Full list of author information is available at the end of the article
}

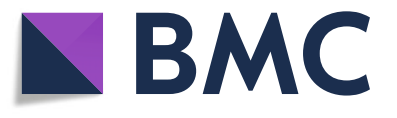

(c) The Author(s). 2022 Open Access This article is licensed under a Creative Commons Attribution 4.0 International License, which permits use, sharing, adaptation, distribution and reproduction in any medium or format, as long as you give appropriate credit to the original author(s) and the source, provide a link to the Creative Commons licence, and indicate if changes were made. The images or other third party material in this article are included in the article's Creative Commons licence, unless indicated otherwise in a credit line to the material. If material is not included in the article's Creative Commons licence and your intended use is not permitted by statutory regulation or exceeds the permitted use, you will need to obtain permission directly from the copyright holder. To view a copy of this licence, visit http://creativecommons.org/licenses/by/4.0/. 
Conclusions: Genetically predicted increase in Vit-D status [25(OH)D] may confer secondary protection against incident combined CVD events and myocardial infarction in a hypertensive-diabetic population where serological 25(OH)D deficiency is common, through facilitating blood pressure control.

Keywords: Vitamin D, Exome Chip, Mendelian randomization, Type 2 diabetes, Hypertension, Secondary prevention, Chinese

\section{Introduction}

Latest Mendelian randomization studies showed that deficiency of vitamin D (Vit-D) may play a causal role in the development of both type 2 diabetes mellitus (T2DM) [1-4] and hypertension [5, 6]. These observations sparkle interests to look for a unifying mechanistic connection. Indeed, T2DM and hypertension frequently co-exist and share intertwined etiology [7-11]. Cardiovascular disease (CVD) represents a major disease burden consequential to uncontrolled hypertension and T2DM. Detrimental effects of hypertension and T2DM exhibit a synergistic effect $[10,11]$. Nevertheless, the upstream etiological origin of hypertension and T2DM remained incompletely understood.

Extensive studies, including large randomized trials [12, 13], showed that Vit-D was unlikely to play a key role in the primary prevention of CVD. However, prior studies had predominantly focused on healthy subjects with normal baseline serum 25-hydroxyvitamin D [(25(OH)D] concentrations $[12,13]$. On the other hand, a protective effect of 25(OH)D against CVD events was noted in highrisk groups, such as diabetic patients [14]. Indeed, increased 25(OH)D was shown in experimental studies to promote vascular regeneration and repair [15]. Its role in the secondary protection of subjects with established CVD or at increased risk was rarely explored.

To test the hypothesis that Vit-D may confer secondary CVD protective effects in the high-risk hypertensivediabetic population, we performed a prospective Mendelian randomization study to investigate any secondary protective effects of serum 25(OH)D on incident CVD endpoints in a cohort of hypertensive subjects with prevailing type 2 diabetes. We further tested a second hypothesis that genetically predicted Vit-D alters CVD outcomes in these hypertensive-diabetic subjects through modulating blood pressure and/or glycemic control.

\section{Research design and methods}

\section{Study subjects}

This study was performed based on the existing platform of the University of Hong Kong Theme-Based Research Scheme Cohort (HKU-TRS), as detailed elsewhere [16]. Briefly, the HKU-TRS recruited a total of 6048 Southern Chinese subjects, who underwent exome chip-genotyping with individual-level quality control. Subjects were prospectively followed up for incident CVD nonfatal and fatal events. Written informed consent was obtained for all subjects. The study has been approved by the Institutional Review Board, Hong Kong West, Hospital Authority, and adhered to the Declaration of Helsinki.

In the current study, the HKU-TRS cohort sample was methodologically structured and analyzed using a framework for two-sample Mendelian randomization (Supplementary Fig. 1).

\section{Derivation subcohort sample}

A total of 1460 subjects (mean age: $56.2 \pm 11.4$ years, $53.8 \%$ male) without hypertension at recruitment constituted a Derivation Subcohort for deriving the genetic instrument for Vit-D pathways for Mendelian randomization. All included subjects had serum 25(OH)D measurements and were genotyped for Vit-D mechanistic pathway genetic variants. Their clinical and biochemical characteristics are presented in Table 1.

\section{Hypertensive-diabetic subcohort sample}

This sample comprised 3746 subjects who had prior history or diagnosis of hypertension per assessment at baseline (mean age: $65.8 \pm 11.6$ years, $62.7 \%$ male), with or without concomitant diabetes mellitus (type 2 diabetes: prevalence $78.9 \%$ ). These subjects represented a sample of heightened underlying cardiovascular risk. The use of lipid-lowering therapy was up to $72.2 \%$. A total of $17.5 \%$ of these subjects had a prior history of any of the following conditions: myocardial infarction, unstable angina, congestive heart failure, ischemic stroke, or peripheral vascular disease. They were prospectively followed up and studied for development of incident hard clinical CVD endpoints. The mean follow-up duration was $55.6 \pm 28.9$ months. The genetic instrumental variable of Vit-D prior obtained from the Derivation Subcohort was independently applied in this sample for causal inference of hard clinical CVD endpoints. To demonstrate consistency of findings, further sensitivity analyses were performed in which the primary analyses were repeated in 2954 subjects who were $100 \%$ co-morbid with hypertension and T2DM.

\section{Baseline demographic, clinical and laboratory assessments}

Baseline demographic data, general cardiovascular risk factors, and definitions of clinical diagnoses were 
Table 1 Derivation and hypertensive-diabetic subcohort sample characteristics, stratified by incident combined cardiovascular (CV) endpoints

\begin{tabular}{|c|c|c|c|c|c|}
\hline & Derivation Subcohort & Hypertensive-Diab & Subcohort & & \\
\hline & (i) $N=1460$ & $\begin{array}{l}\text { Nil incident event } \\
\text { (ii) } N=3185\end{array}$ & $\begin{array}{l}\text { Incident CV endpoints } \\
\text { (iii) } N=561\end{array}$ & & \\
\hline Male $[n(\%)]$ & $786(53.8 \%)$ & $1993(62.6 \%)$ & $354(63.1 \%)$ & $<0.001^{*}$ & 0.81 \\
\hline Age (years) & $56.2 \pm 11.4$ & $64.7 \pm 11.5$ & $71.8 \pm 10.4$ & $<0.001^{*}$ & $<0.001^{*}$ \\
\hline Ever smoking [n (\%)] & $504(34.5 \%)$ & 1167 (36.6\%) & $239(42.6 \%)$ & $0.001^{*}$ & $0.007^{*}$ \\
\hline Body mass index $\left(\mathrm{kgm}^{-2}\right)$ & $24.0 \pm 3.5$ & $25.9 \pm 4.1$ & $25.9 \pm 4.4$ & $<0.001^{*}$ & 0.89 \\
\hline Diabetes mellitus [n (\%)] & $473(32.4 \%)$ & $2485(78.0 \%)$ & $469(83.6 \%)$ & $<0.001^{*}$ & $0.003^{*}$ \\
\hline Systolic blood pressure $(\mathrm{mmHg})$ & $120.0 \pm 14.8$ & $139.3 \pm 19.4$ & $141.4 \pm 21.9$ & $<0.001^{*}$ & $0.031^{*}$ \\
\hline Diastolic blood pressure $(\mathrm{mmHg})$ & $72.1 \pm 8.8$ & $75.5 \pm 11.0$ & $70.1 \pm 11.3$ & $<0.001^{*}$ & $<0.001^{*}$ \\
\hline LDL-cholesterol (mmol/L) & $3.1 \pm 0.9$ & $2.6 \pm 0.9$ & $2.5 \pm 1.0$ & $<0.001^{*}$ & 0.077 \\
\hline HDL-cholesterol (mmol/L) & $1.4 \pm 0.5$ & $1.24 \pm 0.44$ & $1.17 \pm 0.33$ & $<0.001^{*}$ & $<0.001^{*}$ \\
\hline Triglycerides (mmol/L) & $1.4 \pm 1.0$ & $1.6 \pm 1.1$ & $1.6 \pm 1.2$ & $<0.001^{*}$ & 0.66 \\
\hline Fasting glucose (mmol/L) & $6.8 \pm 9.0$ & $7.5 \pm 6.3$ & $8.5 \pm 10.5$ & $0.001^{*}$ & $0.037^{*}$ \\
\hline Creatinine $(\mu \mathrm{mol} / \mathrm{L})$ & $79.8 \pm 50.1$ & $98.4 \pm 74.6$ & $136.6 \pm 113.1$ & $<0.001^{*}$ & $<0.001^{*}$ \\
\hline Lipid-lowering therapy [n (\%)] & $849(58.2 \%)$ & $2254(70.8 \%)$ & $452(80.6 \%)$ & $<0.001^{*}$ & $<0.001^{*}$ \\
\hline 25-hydroxyvitamin $D(\operatorname{Ln})^{\ddagger}$ & $3.02 \pm 0.33$ & $3.01 \pm 0.33$ & $2.98 \pm 0.38$ & $0.035^{*}$ & $0.043^{*}$ \\
\hline Season of recruitment & & & & 0.57 & 0.61 \\
\hline Spring $[n(\%)]$ & $225(15.4 \%)$ & $515(16.2 \%)$ & $88(15.7 \%)$ & & \\
\hline Summer $[n(\%)]$ & $478(32.7 \%)$ & $940(29.5 \%)$ & $178(31.7 \%)$ & & \\
\hline Autumn [n (\%)] & $222(15.2 \%)$ & $537(16.9 \%)$ & $99(17.6 \%)$ & & \\
\hline Winter $[n(\%)]$ & $535(36.6 \%)$ & $1193(37.5 \%)$ & $196(34.9 \%)$ & & \\
\hline Vitamin D GRS & $2.7 \pm 1.2$ & $2.7 \pm 1.2$ & $2.5 \pm 1.3$ & $0.001^{*}$ & $0.002^{*}$ \\
\hline
\end{tabular}

${ }^{*} P<0.05$

${ }^{\dagger} P$ value 1 : Group (iii) versus (i)

$P$ value 2: Group (iii) versus (ii)

${ }^{\ddagger}$ Natural log-transformed due to skewed distribution

'GRS, genetic risk score (linear 0-6) based on allele scoring summation (CYP2R1: rs2060793; GC: rs4588, rs7041)

prior described [16]. Hypertension was defined as either resting systolic/diastolic blood pressure $(\mathrm{BP}) \geq$ $140 / 90 \mathrm{mmHg}$ at two different clinic visits or on medications. For outcome analyses, the JNC-8 guidelines were adopted in which the cut-offs for blood pressure control were systolic $\mathrm{BP}<150 \mathrm{mmHg}$ and diastolic $\mathrm{BP}<90 \mathrm{mmHg}$ (majority of subjects, $68 \%$, were aged $\geq 60$ years) [17]. The American Diabetes Association (ADA) guidelines for blood pressure control targets (systolic < $140 \mathrm{mmHg}$, diastolic < $90 \mathrm{mmHg}$ ) were adopted for subjects with $100 \%$ comorbid hypertension and T2DM [7]. Diabetes mellitus was defined by serum fasting glucose $\geq 7.0 \mathrm{mmol} / \mathrm{L}$ or on medications. Fasting blood was collected for biochemical analysis of serum low-density/high-density lipoprotein (LDL/HDL)-cholesterol, triglycerides, glucose, and creatinine.

\section{Laboratory measurements}

Serum $25(\mathrm{OH}) \mathrm{D}$ concentration was measured using a validated enzyme immunoassay (Abbot, USA). We showed a small inter-assay variability between different validated enzyme immunoassays (correlation coefficient of $0.86, P<0.001$, using Abott, USA versus IDS Diagnostics, UK). We measured serum 25(OH)D in all subjects using the Abbot assay to enhance internal validity. For intra-assay variability, coefficient of variations for serum 25(OH)D ranged between 4.2 and $6.9 \%$ for the low 25(OH)D pool (mean $13.6 \mathrm{ng} /$ $\mathrm{mL}$ [range 11.6-14.8]) and $1.9-2.5 \%$ for the high $25(\mathrm{OH}) \mathrm{D}$ pool (mean $36.8 \mathrm{ng} / \mathrm{mL}$ [range 35.6 to 38.4]) [18], with similar results shown in different populations [19]. Vit-D deficiency was defined by serum $25(\mathrm{OH}) \mathrm{D}<20 \mathrm{ng} / \mathrm{mL}$. Vit-D status was categorized as sufficient $(\geq 30 \mathrm{ng} / \mathrm{mL})$, insufficient $(\geq 20$ to $<30 \mathrm{ng} / \mathrm{mL})$, or deficient $(<20 \mathrm{ng} / \mathrm{mL})$. Seasonality of blood sampling was categorized [16].

\section{Derivation of genetically predicted vitamin D level}

We considered candidate SNPs from various stages of Vit-D mechanistic pathways. Genotyping was performed using a specially designed high-throughput Exome chip 
array (Illumina HumanExome BeadChip, Asian exomechip), as prior described $[20,21]$, to interrogate 12 single-nucleotide polymorphisms (SNPs) involved in the Vit-D mechanistic pathways and detected to have associations at genome-wide or subgenome-wide significance with serum 25(OH)D from prior genetic association or genome-wide associations studies (GWAS) (Biosynthetic: rs4646536, rs10877012, rs3829251, rs1790349; Activation: rs2060793, rs1993116; vitamin D-binding protein (VBP)/GC: rs4588, rs7041, rs2282679, rs1155563; and Vit-D receptor: rs1544410, rs10735810) [22-36]. Prior study supported that a simple unweighted score had similar utility compared to a more complex weighted score in Mendelian randomization analyses [37]. We constructed a simple point-scale 6-alleles genetic risk score (GRS, linear continuous: 0-6), with an approach similar to prior studies [38, 39], based on three SNPs that were significantly associated with serum $25(\mathrm{OH}) \mathrm{D}$ concentration in the Derivation Subcohort. HardyWeinberg equilibrium was tested by $X^{2}$ against each SNP locus in the HKU-TRS cohort to exclude genotypedependent ascertainment bias, using a prior validated equilibrium test calculator [40]. We further excluded linkage disequilibrium (pairwise) by testing against all SNP combinations included in the GRS. A constituent score of 0 to 2 of each SNPs was assigned to the total score according to the allele frequency distribution (AA, $\mathrm{Aa}, \mathrm{aa})$, thus deriving an overall score that ranged from 0 to 6 . The scores of 0 and 6 respectively corresponded to the lowest and highest genetically predicted serum $25(\mathrm{OH}) \mathrm{D}$ concentration.

We obtained Mendelian randomization estimates for the associations of genetically predicted vitamin D status with comprehensive incident CVD events using twosample instrumental variable analysis. Specifically, we obtained SNP-specific Wald's estimates (quotient of genetic association on incident CVD events divided by genetic association on serological Vit-D) and then an overall estimate under an additive genetic model of the GRS (Wald's estimate: $\beta \mathrm{xy}=\beta \mathrm{zy}$ divided by $\beta \mathrm{zx}$ ), and the ratio $95 \%$ confidence interval based upon Fieller's theorem (E.C. Fieller, 1940) [41]. In brief, to meet the three basic assumptions in Mendelian randomization, i.e., including relevance, independence, as well as exclusionsrestriction principle, we employed SNPs that strongly and independently predicted Vit-D levels. We sought to ascertain whether the genetic variants were independent of potential confounders from their crude associations with these variables in the HKU-TRS, including smoking history, use of lipid-lowering therapy, and BMI, and found that there was no significant association (all $P>$ $0.20)$. To strengthen the assumption that such included SNPs were associated with incident CVD events only via serum $25(\mathrm{OH}) \mathrm{D}$, we further checked for known direct effects of the genetic variants on CVD outcomes (i.e., horizontal pleiotropy, thus violating the exclusionsrestriction principle) in three extensive, curated reference databases that recorded well-established associations between genotypes and phenotypes:

i. PhenoScanner (www.phenoscanner.medschl.cam.ac. uk);

ii. GWAS catalog (https://www.ebi.ac.uk/gwas/);

iii. Ensembl (http://www.ensembl.org/index.html);

A search through all these databases as of date of April 2021 found no relation of these SNPs of interests with potential confounders, including tobacco or alcohol use, physical activity, BMI, as well as socioeconomic position/Townsend index. As there was a limited number of SNPs included in our instrument, MR-Egger was not used for reasons that were prior explained [16].

\section{Comprehensive clinical CVD outcomes assessment}

During the prospective follow-up, we adjudicated new onset CV diseases, and CV mortality based on the International Classification of Diseases, Ninth Revision (ICD9), consisting of acute MI (ICD-9 410), unstable angina/ acute coronary syndrome (ACS) (ICD-9 411.1), congestive heart failure (CHF) (ICD-9 428.0), ischemic stroke (ICD-9 433, 434, 435, 436), peripheral vascular disease (PVD) (ICD-9 443.9), and cardiovascular death (death certificate ICD-9 410-447). Data were retrieved and ascertained from the medical records and clinical data network of all public hospitals in Hong Kong, and from directly called back follow-ups.

The primary endpoint was the effect of genetically predicted Vit-D on combined CVD events, defined as occurrence of any of the following: incident MI, ACS, CHF, ischemic stroke, PVD, and CVD death. We defined CVD death as death directly resulting from circulatory disturbances as acute MI, acute/acute-on-chronic heart failure, cardiac arrhythmias, or ischemic/hemorrhagic stroke. Secondary endpoints were the effects of genetically predicted Vit-D on blood pressure and glycemic levels in hypertensive-diabetic subjects, and their impact on combined CVD events.

\section{Statistical analysis}

Relations of serum 25(OH)D with binary clinical outcomes of CVD events and mortality were examined using logistic regression. Variables with skewed distribution were natural log-transformed during analysis. Serum 25(OH)D distribution had significant skewness and was natural log-transformed when appropriate. In the Hypertensive-Diabetic Subcohort, we used logistic and linear regression analyses to assess the relation of serum $25(\mathrm{OH}) \mathrm{D}$ with hypertension control, serum fasting glucose, lipid profile, and body-mass index, as appropriate. Cox proportional hazards regression was used to 
assess the relation of serum 25(OH)D with incident CVD events. Potential confounders were defined based on prior reported associations, specified a priori. Multivariable regression model was used to adjust for potential confounders including age, gender, smoking, diabetes mellitus, lipid-lowering therapy use, body-mass index, systolic/diastolic BP, creatinine, serum LDL/HDL cholesterol/ triglycerides, and seasonal variation of serum $25(\mathrm{OH}) \mathrm{D}$ measurement. To test for linearity assumptions, we repeated analyses with dichotomous models and categorical exposure variables when appropriate. Our study follow-up for primary endpoint had $100 \%$ completion. Any missing values were excluded from analyses.

A two-sided $P$ value less than 0.05 was considered statistically significant. The statistical software packages IBM SPSS (version 21) (SPSS, Chicago, IL), STATA (version 14.0), GraphPad/ PRISM statistical calculators, and R-programming language (version 3.4.3) were used.

\section{Sensitivity analyses}

As our study objective was to investigate the inferred effect of genetically predicted Vit-D on comprehensive clinical CVD events, analyses focused primarily on the constructed instrument GRS (CYP2R1: rs2060793; GC: rs 4588, rs 7041 ) and the primary endpoint. For sensitivity analyses of the genetic instrument, further testing using individual SNPs/their combinations were performed to demonstrate robustness and consistency. As the analyses were based on the constructed Vit-D GRS derived from a priori defined principles, there was no adjustment made for multiple comparisons.

Furthermore, Mendelian randomization outcome analyses in the hypertensive-diabetic subcohort were repeated among the group of subjects with strictly comorbid hypertension and T2DM $(n=2954)$ (Supplementary Fig. 1).

\section{Results}

\section{Serological 25(OH)D deficiency and constructed genetic} instrument

The study flowchart is shown in Supplementary Fig. 1. Baseline clinical characteristics of the Derivation Subcohort and Hypertensive-Diabetic Subcohort are presented in Table 1, the latter stratified by the occurrence of incident combined CVD events. The prevalence of Vit-D deficiency in hypertensive-diabetic subjects (defined as serum $25(\mathrm{OH}) \mathrm{D}<20 \mathrm{ng} / \mathrm{mL})$ was $45.1 \%$.

Based on the Derivation Subcohort, 6 SNPs from two genetic loci implicated in the Vit-D mechanistic pathways (CYP2R1: rs2060793, rs1993116; GC: rs2282679, rs4588, rs1155563, rs7041) were significantly associated with serum 25(OH)D concentration (all $P<0.05$ ). We constructed a GRS based on 3 SNPs with the strongest prediction estimates for 25(OH)D without linkage disequilibrium (CYP2R1: rs2060793; GC: rs4588, rs7041). GRS was independently associated with serum $25(\mathrm{OH}) \mathrm{D}$ ( $\mathrm{B}=0.66$ [95\%CI 0.51 to 0.82 ], $P<0.001$ ). Total variance of absolute serum $25(\mathrm{OH}) \mathrm{D}$ explained by GRS was $2.1 \%$, with F-statistic $=32(P<0.001)$, indicating a strong instrument. Genetic variants included in our instrument showed inter-loci perfect equilibrium $\left(R^{2}=0 \%\right)$. $r s 4588$ and rs7041 demonstrated only weak correlation $\left(R^{2}=\right.$ 0.16). Conveniently, per-allele increase in GRS predicted $15 \%$ reduced risk of Vit-D deficiency (OR $=0.85$ [95\%CI 0.81 to 0.89 ], $P<0.001$, adjusted for age and sex). Tests for Hardy-Weinberg equilibrium revealed no violation (all SNPs $X^{2} 0-1.5$ ) in the HKU-TRS cohort.

Importantly, GRS was here independently derived from the above-mentioned analytic framework. We prior performed another two-sample Mendelian randomization analytic study based on the same HKU-TRS platform using a similar approach, but which specifically focused on a different theme of ischemic vascular events recurrence [16], and yielded a GRS comprising the same constituent SNPs. Such coherent findings substantiate the robustness of predictive utility of this SNPs combination as a validated GRS for serum $25(\mathrm{OH}) \mathrm{D}$.

\section{Protection against combined CVD events in hypertensive- diabetic subjects}

After a mean follow-up duration of $55.6 \pm 28.9$ months, a total of 561 combined CVD events (15\%) had occurred. Specifically, the number of incident events of MI, unstable angina, CHF, ischemic stroke, PVD, and CVD death were 162 (4.3\%), 79 (2.1\%), 372 (9.9\%), 79 (2.1\%), $22(0.6 \%)$, and $35(0.9 \%)$, respectively. Serological $25(\mathrm{OH}) \mathrm{D}$ deficiency $(<20 \mathrm{ng} / \mathrm{mL})$ independently predicted incident combined CVD events ( $\mathrm{HR}=1.3$ [95\%CI 1.1 to 1.5$], P=0.007$, Supplementary Table 2). Conversely, higher Vit-D GRS predicted a lower rate of incident combined CVD events in crude (HR 0.90 [95\%CI 0.84 to 0.97$], P=0.003$ ) and multivariable Cox regression analyses (HR 0.90 [95\%CI 0.84 to 0.96 ], $P=0.002$ ), Fig. 2). Kaplan-Meier analyses showed that lower GRS was associated with worsened event-free survival from combined CVD events (below median: 123.6 [95\%CI 119.8 to 127.5] months, versus $\geq$ median: 131.8 [95\%CI 128.8 to 134.9] months, log-rank $=13.5, P<0.001$, Fig. 1A). Applying two-sample Mendelian randomization, increased genetically predicted Vit-D is protective against combined CVD events in hypertensive subjects with prevailing T2DM (Wald's estimate: $\mathrm{OR}=0.86$ [95\%CI 0.75 to 0.95], Fig. 3). Sensitivity analyses including only subjects with strictly co-morbid hypertension and T2DM yielded similar findings (Wald's estimate: OR $=0.87$ [95\%CI 0.76 to 0.96$])$. 


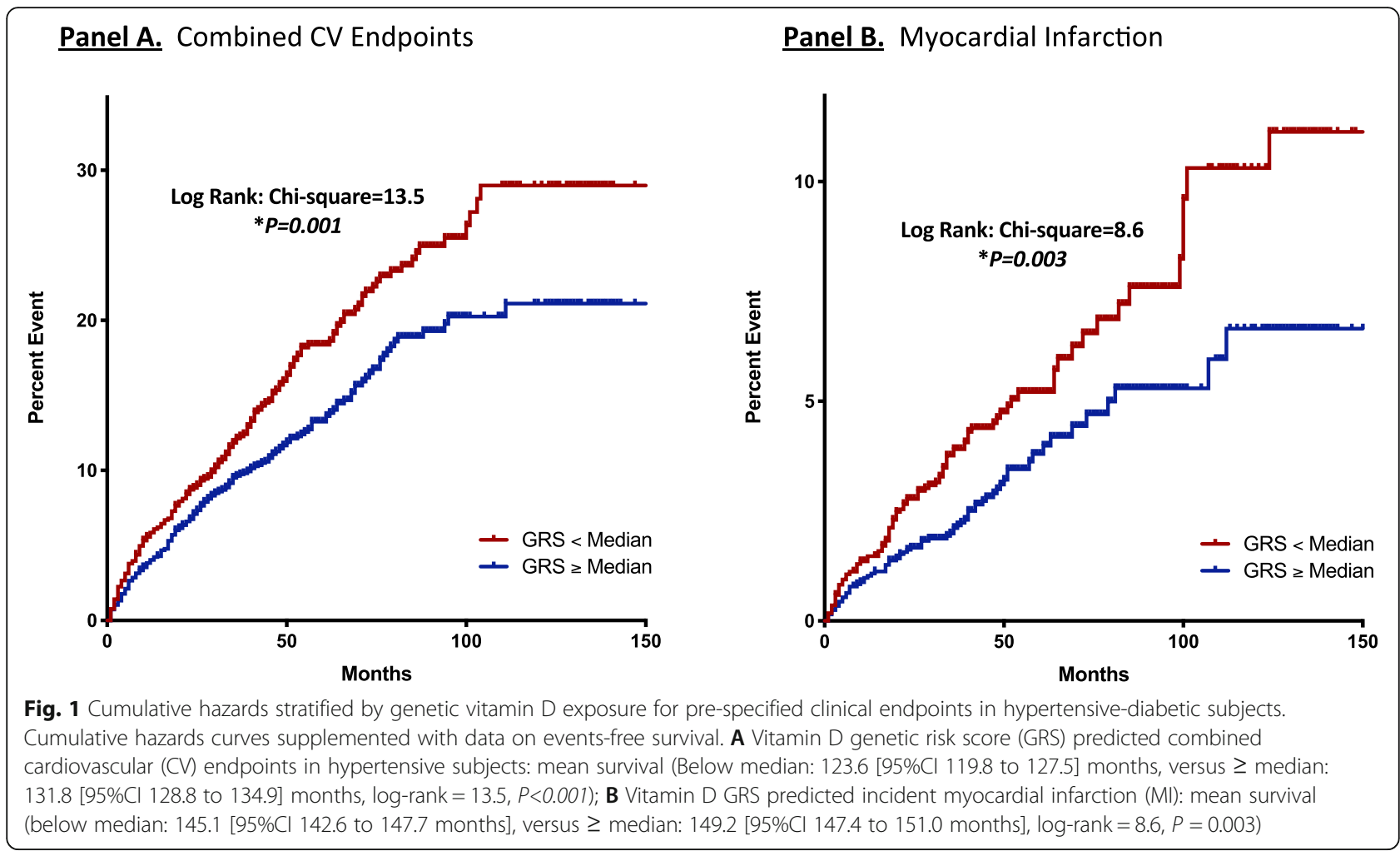

\section{Protection against incident MI in hypertensive-diabetic subjects}

Kaplan-Meier analyses revealed that lower Vit-D GRS was associated with reduced MI event-free survival (below median: 145.1 [95\%CI 142.6 to 147.7 months], versus $\geq$ median: 149.2 [95\%CI 147.4 to 151.0 months], log-rank $=8.6, P=0.003$, Fig. $1 B$ ). Adjusted for potential confounders, per-allele increase in GRS remained independently predictive of reduced new-onset $\mathrm{MI}(\mathrm{HR}=$ 0.83 [95\%CI 0.73 to 0.94 ],$P=0.004$, Fig. 2). Mendelian randomization indicates that increased genetically predicted Vit-D is protective against incident MI (Wald's estimate: $\mathrm{OR}=0.76$ [95\%CI 0.60 to 0.90]). Sensitivity analyses repeated among subjects with strictly comorbid hypertension and T2DM yielded similar findings (Wald' estimate OR $=0.75$ [95\%CI 0.58 to 0.89]).

As shown in Fig. 3, genetically predicted vitamin D status had no significant relations, albeit trends toward negative associations, with other secondary endpoints, including ischemic stroke, CHF, unstable angina, PVD, and CVD death alone in hypertensive-diabetic subjects. Of note, no relations were found between genetic Vit-D exposure and non-CVD death/all-cause mortality.

\section{Mechanistic role of blood pressure control in hypertension and T2DM}

Relations of secondary endpoints, i.e., systolic/diastolic $\mathrm{BP}$ and fasting serum glucose, were examined against serological and genetically predicted 25(OH)D. Systolic $\mathrm{BP}$, serum glucose, triglycerides, and LDL-cholesterol, as well as body-mass index had significant associations with serum $25(\mathrm{OH}) \mathrm{D}$. However, systolic hypertension was the only dependent variable that was negatively predicted by increased GRS (Failed JNC-8 target of systolic $\mathrm{BP}<$ 150 mmHg: $\mathrm{OR}=0.91$ [0.86 to 0.97], $P=0.002$, Supplementary Fig. 2A, Supplementary Table 2). Kaplan-Meier analyses in the Hypertensive-Diabetic Subcohort (4085 subjects, without or without genetic Vit-D measurements) showed that deviation from JNC-8 recommended cut-off for systolic hypertension control was associated with worsened combined CVD endpoints event-free survival (failed JNC-8: 120.0 [95\%CI 114.1 to 126.0 months], versus adhered JNC-8: $131.6 \quad[95 \% \mathrm{CI} \quad 129.2$ to 134.1 months], log-rank $=16.0, P<0.001$, Supplementary Fig. 2B-i). Additionally, such deviation from JNC-8 recommended cut-off also predicted worsened incident MIevent free survival (failed JNC-8: 142.0 [95\%CI 138.2 to 145.9 months] versus Adhered JNC-8: 148.7 [95\%CI 147.1 to 150.2 months], log-rank $=20.5, P<0.001$, not shown in figures).

Mendelian randomization indicates that increased genetically predicted vitamin D status was protective against clinical deviation from achieving JNC-8 target for systolic hypertension control $<150 \mathrm{mmHg}$ (Wald's estimate: $\mathrm{OR}=0.89$ [95\%CI 0.80 to 0.96$]$ ), as well as overall hypertension control (JNC-8: systolic BP $<150 \mathrm{mmHg}$ 


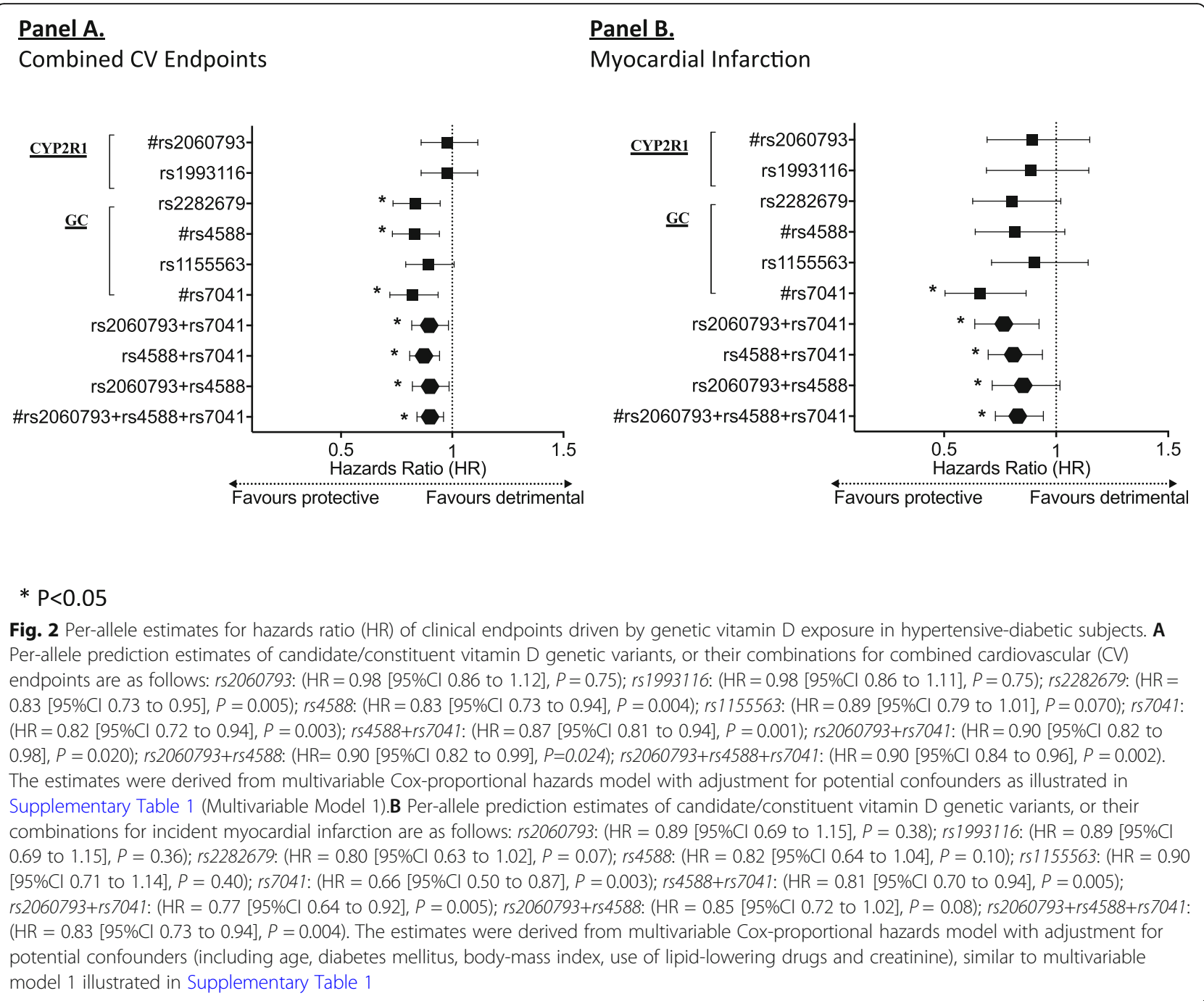

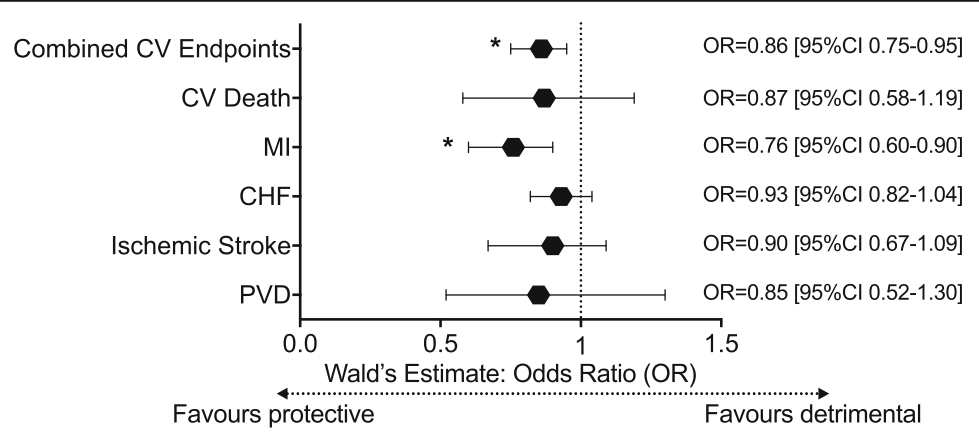

*P<0.05

Fig. 3 Mendelian randomization-inferred causality of vitamin D on incident clinical cardiovascular (CV) endpoints in hypertensive-diabetic subjects. Mendelian randomization showed vitamin D has causally protective effects against incident combined CV endpoints (Wald's estimate: odds ratio $[\mathrm{OR}]=0.86[95 \% \mathrm{Cl} 0.75$ to 0.95$])$ and incident myocardial infarction $(\mathrm{OR}=0.76[95 \% \mathrm{Cl} 0.60$ to 0.90$])$ in 3746 hypertensive subjects. Any causality of vitamin $\mathrm{D}$ on incident $\mathrm{CV}$ death $(\mathrm{OR}=0.87$ [ $95 \% \mathrm{Cl} 0.58$ to 1.19$])$, congestive heart failure $(\mathrm{OR}=0.93$ [95\% $\mathrm{Cl} 0.82$ to 1.04$])$, ischemic stroke $(\mathrm{OR}=0.90[95 \% \mathrm{Cl} 0.67$ to 1.09$])$, and peripheral vascular disease $(\mathrm{OR}=0.85[95 \% \mathrm{Cl} 0.52$ to 1.30$])$ is neither supported nor excluded 
and diastolic $\mathrm{BP}<90 \mathrm{mmHg}$ ) (Wald's estimate: $\mathrm{OR}=$ 0.92 [95\%CI 0.84 to 0.98$]$ ).

\section{Impact of American Diabetes Association guideline- directed systolic hypertension control on CVD outcomes} Analyses repeated among strictly co-morbid hypertensivediabetic subjects $(n=2954)$ showed that deviation from American Diabetes Association (ADA) guideline-directed systolic BP control (control target $<140 \mathrm{mmHg}$ ) predicted worsened combined CVD events-free survival (failed ADA: 124.2 [ $95 \%$ CI 119.5 to 129.0 months] versus adhered ADA: 131.6 [95\%CI 128.3 to 135.0 months], logrank $=11.5, P=0.001$ ). Similarly, deviation from ADA guideline-directed systolic BP control also predicted worsened incident MI-events free survival (failed ADA: 144.9 [95\%CI 142.0 to 147.9 months] versus Adhered ADA: 147.9 [95\%CI 145.7 to 150.2 months], log-rank $=11.7, P=$ 0.001, Supplementary Fig. 2B-ii).

\section{Preferrential protection in Vit-D deficient hypertensive- diabetic subjects}

Each genetically instrumented rise of serum 25(OH)D by $1 \mathrm{ng} / \mathrm{mL}$ confers $14 \%$ reduced odds of combined CVD events in this group of hypertensive-diabetic subjects with prevailing Vit-D deficiency (45.1\%), as well as $8 \%$ reduced odds of deviation from achieving JNC-8 hypertension control. Interestingly, subgroup analyses further revealed that the protective effect of increased vitamin $D$ status against combined CVD events predominated among subjects with Vit-D deficiency (Wald's estimate: $\mathrm{OR}=0.85$ [95\%CI 0.71 to 0.96$]$ ), but was less apparent in those without Vit-D deficiency (OR $=0.90$ [95\%CI 0.77 to 1.03]). The protective effect of Vit-D against failed hypertension control also predominated among hypertensive subjects with Vit-D deficiency (Wald's estimate: $\mathrm{OR}=0.88$ [95\%CI 0.77 to 0.98 ], rather than in those without deficiency $(\mathrm{OR}=0.91 \quad[95 \% \mathrm{CI} \quad 0.80$ to $1.01])$.

\section{Discussion}

To our knowledge, this is the first Mendelian randomization study that investigated vitamin $\mathrm{D}$ status for the focused secondary prevention of CVD events in high-risk persons with hypertension and T2DM.

Hypertension is two-fold as common in patients with T2DM, with prevalence estimates varying between 74 and $85 \%[8,9]$. Conversely, hypertensive subjects exhibit greater preponderance of insulin resistance than normotensive persons. The conjunctive presence of hypertension and T2DM exhibits synergistic effects in escalating CVD risks $[10,11]$. Thus optimizing CVD risk control in this vulnerable group of hypertensive-diabetic subjects is of paramount importance in their clinical care. Vit-D deficiency is pandemic and highly reversible. Given its causal associations found with both T2DM [1-4] and hypertension [5, 6] in latest Mendelian randomization studies, it is imperative to know whether this further translates into clinically manifest CVD events and the underlying mechanisms.

Findings from our study indicated that a genetically predicted increase in serum $25(\mathrm{OH}) \mathrm{D}$ is protective against combined incident CVD events, incorporating CVD death, in these high-risk hypertensive-diabetic subjects. It is also protective against incident MI. Moreover, we further showed that genetically predicted Vit-D attenuates systolic hypertension and is a driving force for achieving guideline-directed blood pressure control in persons with T2DM and hypertension, resulting in reduced CVD events. Together with our recent Mendelian randomization focused analyses showing that genetically predicted increase in serum 25(OH)D protects against the clinical recurrence of ischemic stroke and myocardial infarction [16], the benefits of increased serum $25(\mathrm{OH}) \mathrm{D}$ appear to be conferred to high-risk subjects in the advanced spectrum of the cardiovascular continuum.

Our finding of genetically predicted higher serum $25(\mathrm{OH}) \mathrm{D}$ having ameliorating effects on systolic hypertension is consistent with prior literature. In a large meta-analyzed Mendelian randomization on hypertension, each $10 \%$ of genetically instrumented increase in serum $25(\mathrm{OH}) \mathrm{D}$ was causally associated with $8.1 \%$ decreased odds of hypertension [6]. Another large Danish prospective Mendelian randomization study subsequently showed similar findings [5]. Importantly, we made a solid step further and showed that better vitamin D status facilitated adherence to guideline-directed blood pressure control in hypertensive-diabetic subjects. This in turn translated into secondary reduction in CVD endpoints. Nevertheless, while prior Mendelian randomization studies supported a causal role of predicted decreases in serum $25(\mathrm{OH}) \mathrm{D}$ on the development of type 2 diabetes [3, 4], our data did not find a relation of genetically predicted Vitamin D status on fasting glucose level. The reason for this was unclear. The lack of current mechanistic exploration specifically on insulin resistance, a common condition linked to hypertension, is a limitation of our study. Prior Mendelian randomization studies on specific cardiovascular risk factors corroborate our findings of no significant effects of predicted changes in serum $25(\mathrm{OH}) \mathrm{D}$ on lipid profile [42].

The reason why predicted increases in serum 25(OH)D may lead to improved blood pressure control is at present unclear. Mechanistically, a lack of renin suppression that is associated with Vit-D deficiency may exacerbate hypertension. Other than facilitating hypertension control, there are several mechanisms through which Vit-D may confer secondary protection in highrisk hypertensive-diabetic subjects. Experimental studies showed that Vit-D promotes angiogenic myeloid cells 
homing to injured vasculature via stromal cell-derived factor (SDF1), enhancing re-endothelialization and vascular regeneration [43]. It also modulates activity of circulating endothelial progenitor cells [44] and macrophages [45], with a potential effect of retarding cellular senescence [46].

Our study has several important strengths. Firstly, given the effects of Vit-D on vascular regeneration and repair [43], our study hypothesis specifically focused on the secondary prevention of high-risk subjects with hypertension and T2DM. This area of secondary protection has been largely unexplored by prior studies. Secondly, this is a prospective Mendelian randomization study with, to our knowledge, the most comprehensive interrogation of incident CVD hard clinical endpoints, incorporating CVD death. Thirdly, our high-throughput exome-chip screened 12 candidate SNPs from comprehensive Vit-D mechanistic pathways and derived a GRS with $\mathrm{R}^{2}$ of $2.1 \%$ and Fstatistic of 32, which has a good explanatory effect size for serum 25(OH)D among Mendelian randomization studies focusing on cardiac outcomes with equivalent sample size or above [38, 39, 47]. Despite large sample sizes, genetic instruments used in the prior several Mendelian randomization studies had typically $R^{2}$ for variance of serum 25(OH)D less than, or at best equal to $1.0 \%$ [38, 39]. Furthermore, we used individual data rather than pooled summary statistics. Prior GWAS revealed that variance of predicted changes in serum $25(\mathrm{OH}) \mathrm{D}$ was explained by genetic variants of Vit-D pathways by no more than $5 \%$ [30]. It is known that Mendelian randomization analyses using two-sample summary statistics are preferentially biased toward null especially in the case of a weak instrument [48], which could be exactly the case with VitD. Fourthly, our prospective follow-up rate of $100 \%$ with high incident event rates (15\% primary endpoints over 5 years) maximized the cohort's ability to detect any differences in CVD outcomes in hypertensive-diabetic subjects. Fifthly, the $45 \%$ prevalence of Vit-D deficiency in our hypertensive-diabetic sample provided excellent variability in serological Vit-D status for the study of causal inference.

Nonetheless, the choice of SNPs included in this investigation was limited by literature available at the time of study conception. Subsequent studies had identified further important genetic variants [49], constituting a limitation of our study. While acknowledging that a possibility of residual biases or confounding in either directions, or presence of horizontal pleiotropy cannot be completely excluded, we carefully studied each included SNP (rs2060793, rs4588, rs7041) in three extensive reference genetic databases. We found that none of the genetic variants included was related to key potential confounders such as tobacco or alcohol use, physical activity, BMI, as well as socioeconomic position.

\section{Conclusions}

We conclude that increased genetically predicted serum $25(\mathrm{OH}) \mathrm{D}$ has a secondary protective effect against incident combined CVD events and MI in high-risk Chinese subjects with hypertension and T2DM. Such effects are mediated at least partially through ameliorated hypertension, and predominantly benefit those with serologically 25(OH)D deficiency (Supplementary Figure 3).

\section{Supplementary Information}

The online version contains supplementary material available at https://doi. org/10.1186/s12263-022-00704-z.

\begin{abstract}
Additional file 1: Supplementary Figure 1. Study Flowchart. Study flowchart detailing the HKU-TRS Cohort, with the methodological framework of Derivation Subcohort $(n=1460)$ and the Hypertensive-Diabetic Subcohort ( $n=3746$ ) samples that underwent two-sample Mendelian randomization for causal inference of Vitamin D on prospective cardiovas cular endpoints in hypertensive subjects with prevailing type 2 diabetes. Supplementary Figure 2. Panel A. Serological and Genetic Vitamin D Prediction Risk Estimates for Deviation from Achieving Guideline-Directed Systolic Hypertension Control. Adjusted for sex and age, per-standard deviation increase in serum 25-hydroxyvitamin D and per-allele increase in Genetic Risk Score (GRS) respectively predicted 11\% (OR=0.89 [95\%Cl 0.83 to 0.96 ], $P=0.002)$ and $9 \%(\mathrm{OR}=0.91$ [0.86 to 0.97 ], $P=0.002)$ reduced odds of deviation from JNC-8 guideline-recommended target for systolic hypertension (systolic <150mmHg). (PDF 398 kb) Panel B. (i). Failed JNC-8 Guideline Cut-off For Systolic Hypertension Predicts Risk of Combined Cardiovascular (CV) Events. Kaplan-Meier analysis showing deviation from JNC-8 recommended treatment target for systolic hypertension $(<$ $150 \mathrm{mmHg}$ ) was associated with worsened combined CV endpoints event-free survival $(120.0$ [95\%Cl 114.1 to 126.0 months] versus 131.6 [95\%Cl 129.2 to 134.1 months], log-rank=16.0, $P<0.001$ ); (ii). Failed ADA Guideline Cut-off For Systolic Hypertension Predicts Risk of Combined CV Events. Deviation from ADA guideline-directed systolic BP control (contro target $<140 \mathrm{mmHg}$ ) predicted worsened combined CVD events-free survival $(124.2[95 \% \mathrm{Cl} 119.5$ to 129.0 months] versus $131.6[95 \% \mathrm{Cl} 128.3$ to 135.0 months], log-rank=11.5, $P=0.001$ ). Supplementary Figure 3 . Theoretical Paradigm. Two-sample Mendelian randomization supports causality of Vitamin D protecting against incident combined CVD events and myocardial infarction in hypertensive-diabetic subjects, at least partially through facilitating guideline-directed clinical control of blood pressure.
\end{abstract}

Additional file 2: Supplementary Table 1. Serological and Genetic Vitamin D Exposure in Prediction Models for Combined Cardiovascular (CV) Endpoints in Hypertensive-Diabetic Subjects ${ }^{+}$.

Additional file 3: Supplementary Table 2. Prediction estimates of VitD GRS $^{\llcorner}$for cardiovascular (CV) Risk Factors ${ }^{\dagger}$

Acknowledgements

Not applicable.

\section{Authors' contributions}

Y.H.C. originated the research hypothesis and conceptual design, secured proposal grant funding, analyzed the data, and wrote the manuscript. J.J.H., Y.K.W., K.W.A., C.S.T., and C.Y.C. collected the data and wrote the manuscript; C.M.S., S.L.A.Y., and J.Z. performed critical statistical analyses and wrote the manuscript; G.N.T., K.K.C., and C.Q.J. provided critical input in design of study methods and conceptualization, and wrote the manuscript; A.X. and S.P.C. provided critical inputs in study methods, and molecular/genetic analyses, and wrote the manuscript; T.H.L., K.S.L., and H.F.T. supervised the overall research conceptualization and direction, study design and implementation, data collection and analysis, cohort supervision, and writing of manuscript, and are being responsible for the overall content as guarantors. All authors revised and approved the submission. 


\section{Funding}

This study was funded by the Health and Medical Research Fund, HKSAR (Project no. 10111531) and the Hong Kong Research Grants Council ThemeBased Research Scheme (T12-705/11), General Research Fund (777511M, 776412M, 776513M and 17128515) and Innovation and Technology Support Programme (Tier 3) (ITS/303/ 12). Y.H.C. was supported by the Li Shu Fan Medical Fellowship for Internal Medicine, The University of Hong Kong.

\section{Availability of data and materials}

Data generated during this study will not be made publicly available, because the act of data sharing had not been incorporated as part of the original study protocol at the time of Ethics Committee review and approval. The authors have given the best efforts in presenting the comprehensive and relevant data in the current manuscript.

\section{Declarations}

Ethics approval and consent to participate

The study was approved by the Institutional Review Board, Hong Kong West, Hospital Authority. All participants gave informed consent.

\section{Consent for publication}

No individually identifiable data was included in this manuscript.

\section{Competing interests}

The authors declare no competing interests.

\section{Author details}

'Division of Cardiology, Queen Mary Hospital, The University of Hong Kong, Hong Kong, China. ${ }^{2}$ School of Public Health, The University of Hong Kong, Hong Kong, SAR, China. ${ }^{3}$ Department of Medicine, Shenzhen Hong Kong University Hospital, Shenzhen, China. ${ }^{4}$ Department of Public Health and Epidemiology, University of Birmingham, Birmingham, UK. ${ }^{5}$ Guangzhou No. 12 Hospital, Guangzhou, People's Republic of China. ${ }^{6}$ Department of Psychiatry and Centre for Genomic Sciences, University of Hong Kong, Hong Kong, China. ${ }^{7}$ Division of Endocrinology, Queen Mary Hospital, The University of Hong Kong, Hong Kong, China. ${ }^{8}$ Hong Kong-Guangdong Joint Laboratory on Stem Cell and Regenerative Medicine, The University of Hong Kong, Hong Kong, China. 'Shenzhen Institutes of Research and Innovation, The University of Hong Kong, Hong Kong, SAR, China.

Received: 17 July 2021 Accepted: 18 January 2022

Published online: 29 January 2022

\section{References}

1. Wang M, Zhou T, Li X, et al. Baseline Vitamin D Status, sleep patterns, and the risk of incident type 2 diabetes in data from the UK biobank study. Diabetes Care. 2020:43(11):2776-84.

2. Dawson-Hughes B, Staten MA, Knowler WC, et al. Intratrial exposure to vitamin $\mathrm{D}$ and new-onset diabetes among adults with prediabetes: a secondary analysis from the vitamin D and type 2 diabetes (D2d) Study. Diabetes Care. 2020

3. Lu L, Bennett DA, Millwood IY, et al. Association of vitamin D with risk of type 2 diabetes: a Mendelian randomisation study in European and Chinese adults. PLoS Med. 2018;15(5):e1002566.

4. Yuan S, Jiang X, Michaelsson K, Larsson SC. Genetic prediction of serum 25hydroxyvitamin D, calcium, and parathyroid hormone levels in relation to development of type 2 diabetes: a mendelian randomization study. Diabetes Care. 2019;42(12):2197-203.

5. Afzal S, Nordestgaard BG. Vitamin D, Hypertension, and ischemic stroke in 116655 individuals from the general population: a genetic study. Hypertension. 2017.

6. Vimaleswaran KS, Cavadino A, Berry DJ, et al. Association of vitamin D status with arterial blood pressure and hypertension risk: a mendelian randomisation study. Lancet Diabetes Endocrinol. 2014;2(9):719-29.

7. de Boer $\mathrm{IH}$, Bangalore S, Benetos A, et al. Diabetes and hypertension: a position statement by the American Diabetes Association. Diabetes Care 2017;40(9):1273-84.

8. Passarella P, Kiseleva TA, Valeeva FV, Gosmanov AR. Hypertension Management in Diabetes: 2018 Update. Diabetes Spectr. 2018;31(3):218-24.
9. Petrie JR, Guzik TJ, Touyz RM. Diabetes, hypertension, and cardiovascular disease: clinical insights and vascular mechanisms. Can J Cardiol. 2018;34(5): 575-84.

10. Gosmanov AR, Lu JL, Sumida K, et al. Synergistic association of combined glycemic and blood pressure level with risk of complications in US veterans with diabetes. J Hypertens. 2016;34(5):907-13.

11. Long AN, Dagogo-Jack S. Comorbidities of diabetes and hypertension: mechanisms and approach to target organ protection. J Clin Hypertens (Greenwich). 2011;13(4):244-51.

12. Scragg R, Stewart AW, Waayer D, et al. Effect of monthly high-dose vitamin D supplementation on cardiovascular disease in the vitamin D assessment study: a randomized clinical trial. JAMA Cardiol. 2017;2(6):608-16.

13. Manson JE, Cook NR, Lee IM, et al. Vitamin D supplements and prevention of cancer and cardiovascular disease. N Engl J Med. 2018.

14. Wan Z, Guo J, Pan A, Chen C, Liu L, Liu G. Association of serum 25hydroxyvitamin $D$ concentrations with all-cause and cause-specific mortality among individuals with diabetes. Diabetes Care. 2020.

15. Witte KK, Byrom R, Gierula J, et al. Effects of vitamin D on cardiac function in patients with chronic HF: the VINDICATE study. J Am Coll Cardiol. 2016; 67(22):2593-603.

16. Chan $\mathrm{YH}$, Schooling $\mathrm{CM}$, Zhao J, et al. Mendelian randomization focused analysis of vitamin $\mathrm{D}$ on the secondary prevention of ischemic stroke. Stroke. 2021:52(12):3926-37.

17. James PA, Oparil S, Carter BL, et al. 2014 evidence-based guideline for the management of high blood pressure in adults: report from the panel members appointed to the Eighth Joint National Committee (JNC 8). JAMA. 2014:311(5):507-20.

18. Farrell CJ, Martin S, McWhinney B, Straub I, Williams P, Herrmann M. Stateof-the-art vitamin $D$ assays: a comparison of automated immunoassays with liquid chromatography-tandem mass spectrometry methods. Clin Chem. 2012;58(3):531-42.

19. Koivula MK, Matinlassi N, Laitinen P, Risteli J. Four automated $25-\mathrm{OH}$ total vitamin D immunoassays and commercial liquid chromatography tandemmass spectrometry in Finnish population. Clin Lab. 2013:59(3-4):397-405.

20. Tang CS, Zhang $\mathrm{H}$, Cheung CY, et al. Exome-wide association analysis reveals novel coding sequence variants associated with lipid traits in Chinese. Nat Commun. 2015;6:10206.

21. Cheung CYY, Tang CS, Xu A, et al. An exome-chip association analysis in Chinese subjects reveals a functional missense variant of GCKR that regulates FGF21 levels. Diabetes. 2017;66(6):1723-8.

22. Bailey R, Cooper JD, Zeitels $L$, et al. Association of the vitamin D metabolism gene CYP27B1 with type 1 diabetes. Diabetes. 2007;56(10):2616-21.

23. Gong $C$, Long $Z$, Yu Y, et al. Dietary factors and polymorphisms in vitamin D metabolism genes: the risk and prognosis of colorectal cancer in northeast China. Sci Rep. 2017;7(1):8827.

24. Signorello LB, Shi J, Cai $Q$, et al. Common variation in vitamin D pathway genes predicts circulating 25-hydroxyvitamin D Levels among African Americans. PLoS ONE. 2011;6(12):e28623.

25. Waldenstrom J, Nystrom K, Nilsson S, et al. The relation of 25-hydroxy vitamin $\mathrm{D}$ concentrations to liver histopathology, seasonality and baseline characteristics in chronic hepatitis C virus genotype 2 or 3 infection. PLoS ONE. 2020;15(8):e0237840.

26. Shaat N, Katsarou A, Shahida B, Prasad RB, Kristensen K, Planck T. Association between the rs1544410 polymorphism in the vitamin D receptor (VDR) gene and insulin secretion after gestational diabetes mellitus. PLOS ONE. 2020;15(5):e0232297.

27. Ahn J, Yu K, Stolzenberg-Solomon R, et al. Genome-wide association study of circulating vitamin D levels. Hum Mol Genet. 2010;19(13):2739-45.

28. Abu El Maaty MA, Hassanein SI, Sleem HM, Gad MZ. Effect of polymorphisms in the NADSYN1/DHCR7 locus (rs12785878 and rs1790349) on plasma 25-hydroxyvitamin D levels and coronary artery disease incidence. J Nutrigenet Nutrigenomics. 2013;6(6):327-35.

29. Robien K, Butler LM, Wang R, et al. Genetic and environmental predictors of serum 25-hydroxyvitamin D concentrations among middle-aged and elderly Chinese in Singapore. Br J Nutr. 2013;109(3):493-502

30. Wang TJ, Zhang F, Richards JB, et al. Common genetic determinants of vitamin D insufficiency: a genome-wide association study. Lancet. 2010; 376(9736):180-8.

31. Enlund-Cerullo M, Koljonen L, Holmlund-Suila E, et al. Genetic variation of the vitamin D binding protein affects vitamin $D$ status and response to supplementation in infants. J Clin Endocrinol Metab. 2019;104(11):5483-98. 
32. Kwak SY, Cho Y, Oh H, Shin MJ. Association of circulating 25-hydroxyvitamin $D$ levels with hypertension and blood pressure values in Korean adults: a Mendelian randomization study on a subset of the Korea National Health and Nutrition Survey 2011-2012 population. Nutr Res Pract. 2019;13(6):498508.

33. Zhao Y, Liao S, He J, et al. Association of vitamin D receptor gene polymorphisms with metabolic syndrome: a case-control design of population-based cross-sectional study in North China. Lipids Health Dis. 2014;13:129.

34. Ma Y, Xiang $Z$, Yao $X$, et al. Associations between vitamin $D$ receptor gene polymorphisms and chronic spontaneous urticaria in Chinese Han population. Postepy Dermatol Alergol. 2020;37(2):250-4.

35. Al-Ghafari AB, Balamash KS, Al Doghaither HA. Relationship between Serum Vitamin D and Calcium Levels and Vitamin D Receptor Gene Polymorphisms in Colorectal Cancer. Biomed Res Int. 2019;2019:8571541.

36. Smolders J, Damoiseaux J, Menheere P, Tervaert JW, Hupperts R. Fok-1 vitamin D receptor gene polymorphism (rs10735810) and vitamin D metabolism in multiple sclerosis. J Neuroimmunol. 2009;207(1-2):117-21.

37. Burgess S, Thompson SG. Use of allele scores as instrumental variables for Mendelian randomization. Int J Epidemiol. 2013;42(4):1134-44.

38. Afzal S, Brondum-Jacobsen P, Bojesen SE, Nordestgaard BG. Genetically low vitamin D concentrations and increased mortality: Mendelian randomisation analysis in three large cohorts. BMJ. 2014;349:g6330.

39. Brondum-Jacobsen P, Benn M, Afzal S, Nordestgaard BG. No evidence that genetically reduced 25 -hydroxyvitamin $D$ is associated with increased risk of ischaemic heart disease or myocardial infarction: a Mendelian randomization study. Int J Epidemiol. 2015;44(2):651-61.

40. Rodriguez S, Gaunt TR, Day IN. Hardy-Weinberg equilibrium testing of biological ascertainment for Mendelian randomization studies. Am J Epidemiol. 2009;169(4):505-14.

41. Au Yeung SL, Luo S, Schooling CM. The impact of glycated hemoglobin (HbA1c) on cardiovascular disease risk: a mendelian randomization study using UK Biobank. Diabetes Care. 2018:41(9):1991-7.

42. Ooi EM, Afzal S, Nordestgaard BG. Elevated remnant cholesterol in 25hydroxyvitamin D deficiency in the general population: Mendelian randomization study. Circ Cardiovasc Genet. 2014;7(5):650-8.

43. Wong MS, Leisegang MS, Kruse $C$, et al. Vitamin D promotes vascular regeneration. Circulation. 2014;130(12):976-86.

44. Chan YH, Lau KK, Yiu KH, et al. Vascular protective effects of statin-related increase in serum 25-hydroxyvitamin D among high-risk cardiac patients. Cardiovasc Med (Hagerstown). 2015;16(1):51-8.

45. Oh J, Weng S, Felton SK, et al. 1,25(OH)2 vitamin d inhibits foam cell formation and suppresses macrophage cholesterol uptake in patients with type 2 diabetes mellitus. Circulation. 2009;120(8):687-98.

46. Richards JB, Valdes AM, Gardner JP, et al. Higher serum vitamin D concentrations are associated with longer leukocyte telomere length in women. Am J Clin Nutr. 2007;86(5):1420-5.

47. Manousaki D, Mokry LE, Ross S, Goltzman D, Richards JB. Mendelian randomization studies do not support a role for vitamin $\mathrm{D}$ in coronary artery disease. Circ Cardiovasc Genet. 2016;9(4):349-56.

48. Haycock PC, Burgess S, Wade KH, Bowden J, Relton C, Davey SG. Best (but oft-forgotten) practices: the design, analysis, and interpretation of Mendelian randomization studies. Am J Clin Nutr. 2016;103(4):965-78.

49. Revez JA, Lin T, Qiao Z, et al. Genome-wide association study identifies 143 loci associated with 25 hydroxyvitamin D concentration. Nat Commun. 2020;11(1):1647.

\section{Publisher's Note}

Springer Nature remains neutral with regard to jurisdictional claims in published maps and institutional affiliations.

Ready to submit your research? Choose BMC and benefit from:

- fast, convenient online submission

- thorough peer review by experienced researchers in your field

- rapid publication on acceptance

- support for research data, including large and complex data types

- gold Open Access which fosters wider collaboration and increased citations

- maximum visibility for your research: over $100 \mathrm{M}$ website views per year

At BMC, research is always in progress.

Learn more biomedcentral.com/submissions 